\title{
Lasers à excimères de forte puissance moyenne à grande cadence
}

\author{
B. Lacour, H. Besaucele, H. Brunet, C. Gagnol et B. Vincent \\ LASERDOT, Groupe Aerospatiale, Route de Nozay, 91460 Marcoussis, France
}

\section{RESUME}

Dans le cadre d'un programme EUREKA EU 205, LASERDOT développe depuis plusieurs années un laser à excimères de grande puissance moyenne fonctionnant à haute cadence de répétition. Une première maquette a été réalisée. Ses performances à $308 \mathrm{~nm}$ correspondent bien aux objectifs visés : énergie par impulsion jusqu'à $1,4 \mathrm{~J}$, cadence de répétition jusqu'à $700 \mathrm{~Hz}$ et puissance moyenne de l'ordre de $500 \mathrm{~W}$. Les études récentes ont porté sur l'amélioration de la divergence qui peut, même à haute cadence, être inférieure à $10^{-4} \mathrm{rad}$.

De cette maquette a été dérivé un prototype industriel qui reprend une bonne partie des solutions techniques déjà étudiées mais sous une forme plus compacte. Ce laser actuellement en cours de tests est prévu pour délivrer de façon fiable et continue une puissance de $500 \mathrm{~W}$ moyens à $500 \mathrm{~Hz}$. Son volume total est de $1,3 \times 1,80 \times 2 \mathrm{~m}$. Ce volume comprend aussi les pompes, les bouteilles de gaz, les alimentations et l'électronique de contrôle. Les premiers résultats sont tout à fait satisfaisants. L'énergie par tir atteint $2 \mathrm{~J}$ et le rendement intrinsèque est supérieur à $4 \%$, tout ceci à $308 \mathrm{~nm}$. A $500 \mathrm{~Hz}$, ce prototype peut délivrer jusqu'à $750 \mathrm{~W}$ moyens.

\section{INTRODUCTION}

Certes, les lasers à excimères possèdent des propriétés extrêmement attirantes en matière de longueurs d'onde, d'énergie, de puissance crête ou de rendement. Pourtant, ils n'ont pas été jusqu'ici beaucoup utilisés dans l'industrie. Il y a a cela deux raisons :

- d'une part, à cause de leur faible taux de récurrence et leur faible puissance moyenne, il n'était pas envisageable de les utiliser pour des applications à productivité élevée ; 
- d'autre part, on leur reprochait, parfois de façon injustifiée d'ailleurs, une fiabilité insuffisante.

Depuis plusieurs années, LASERDOT est impliqué dans le programme EUREKA EU 205 dont le but est de proposer à terme un ou des lasers à excimères industriels d'une puissance moyenne voisine du $\mathrm{kW}$.

Dans ce cadre, LASERDOT s'est orienté plus particulièrement vers la conception et la réalisation de lasers photodéclenchés fonctionnant à relativement haute cadence de répétition : $500 \mathrm{~Hz}$ ou plus avec une énergie par tir modérée : de l'ordre de 1 à 2 Joules.

On le sait, les lasers à excimères utilisent, pour leur circuits de conmutation, des thyratrons qui sont soumis à des contraintes électriques sévères. C'est ce qui explique en grande partie la fiabilité insuffisante de certains de ces appareils.

C'est pourquoi, LASERDOT utilise la technique du photodéclenchement ${ }^{1}$ qui permet, en réduisant le courant crête subit par le thyratron principal d'en accroître considérablement la durée de vie.

Cette technique, que LASERDOT maîtrise depuis longtemps sur des lasers à faible taux de répétition, a d'abord été testée sur la maquette 530 dont nous donnons les caractéristiques et les performances plus loin. A partir de ces expériences, nous avons conçu et réalisé le premier prototype 635 destiné à être commercialisé. Nous en indiquerons plus loin les caractéristiques et performances.

\section{DESCRIPTION DES LASERS}

\subsection{Maquette $\mathbf{5 3 0}$}

Ce laser a déjà été décrit précédemment 2,3 . Ses caractéristiques principales sont les suivantes: volume de décharge $50 \mathrm{~cm} \times 3 \mathrm{~cm} \times 3 \mathrm{~cm}$, tension de charge : jusqu'à $28 \mathrm{kV}$, énergie stockée dans les condensateurs : jusqu'à $60 \mathrm{~J}$, vitesse du gaz entre les électrodes : $30 \mathrm{~m} / \mathrm{s}$ à 5 bars. La figure 1 montre ce laser vue en coupe. On remarquera la compacité de l'ensemble de la boucle de circulation de gaz. Rappelons le, ce laser a été conçu comme un banc de test des divers choix scientifiques et technologiques. De fait, il comporte un certain nombre de dispositions originales : tête à isolant céramique mince, cathode en matériau réfractaire, passages toumant étanches par exemple.

\subsection{Prototype 635}

Les essais sur la maquette 530 ayant donné satisfaction, nous avons décidé de réaliser le prototype d'un laser industriel de $500 \mathrm{~W}$. Nous avons repris pour l'essentiel, les choix technologiques et scientifiques de la maquette. Une nouvelle disposition des circuits de puissance a toutefois permis d'améliorer l'uniformité du champ électrique entre les électrodes et de doubler le volume de décharge tout en conservant le même volume total.

Comme on peut le constater sur la figure 2 , ce laser comporte une partie active placée à l'intérieur d'un cylindre en acier inoxydable d'environ $1 \mathrm{~m}$ de diamètre et $1 \mathrm{~m}$ de long. Les moteurs, alimentations à haute tension, bouteilles de gaz et circuit de contrôle et de commande sont disposés autour de ce cylindre et forment ainsi un ensemble compact $\left(1,3 \times 1,8 \times 2 \mathrm{~m}^{3}\right)$ blindé et insonorisé. 


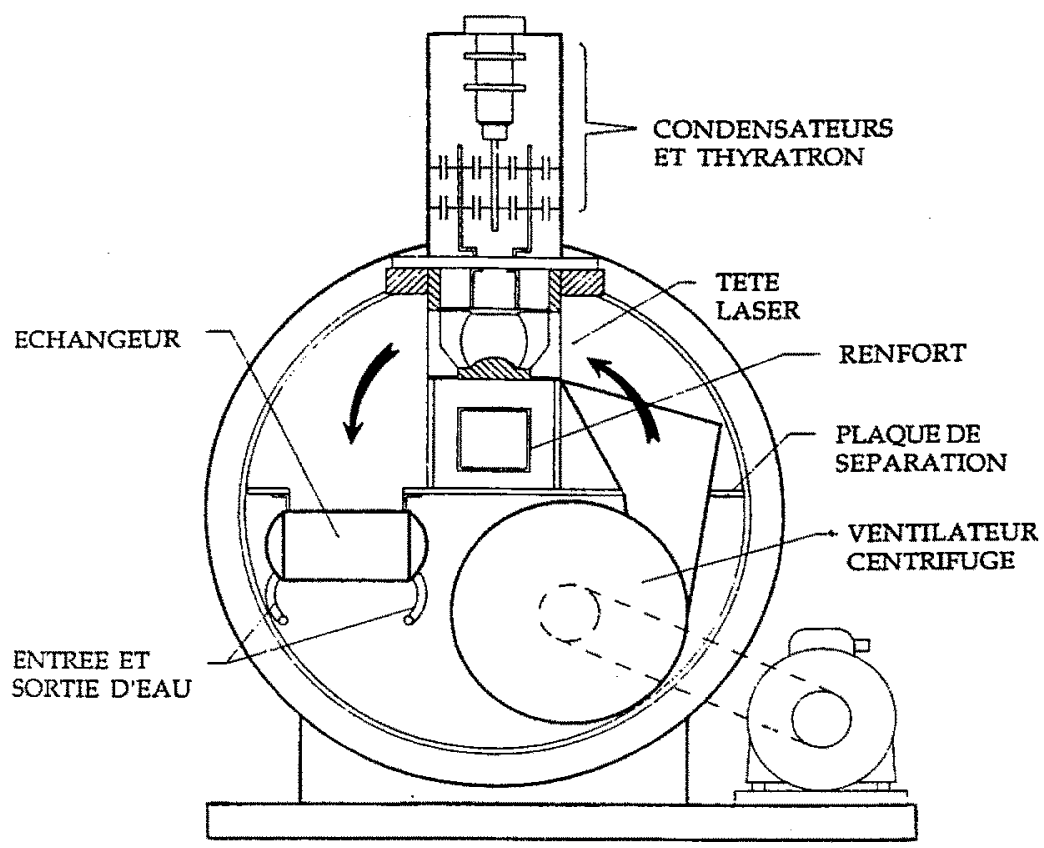

Figure 1 : Vue en coupe de la maquette 530

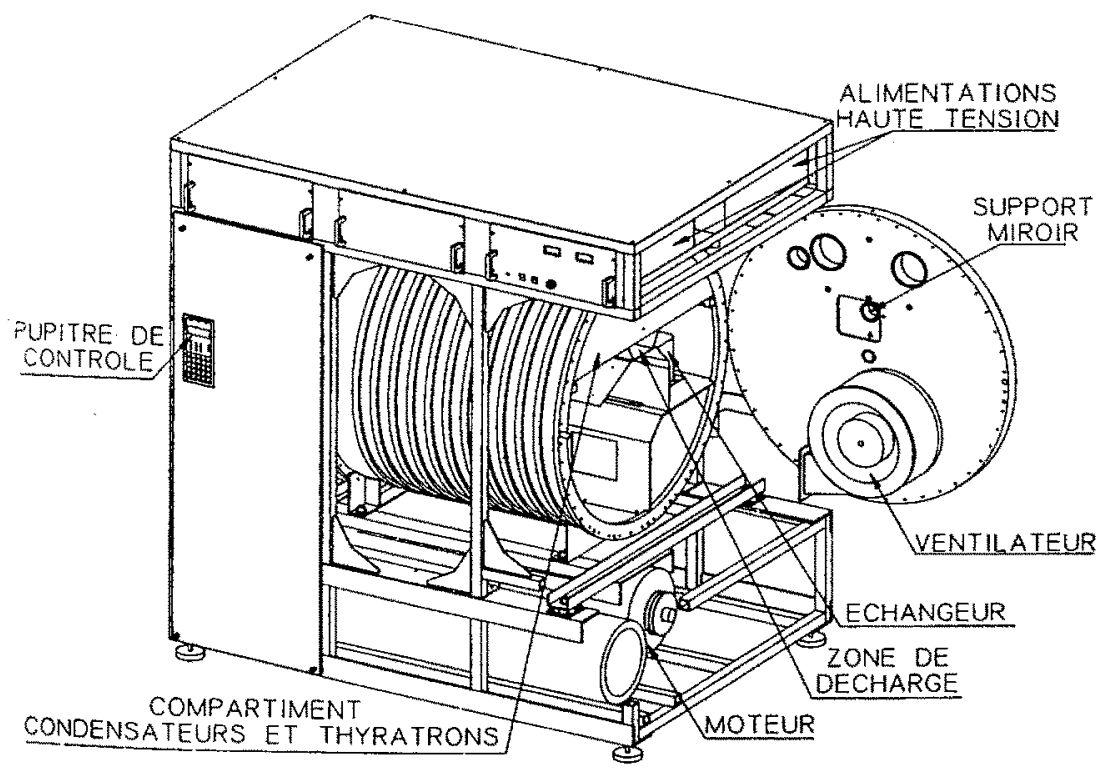

Figure 2: Vue du prototype 635 ouvert 
De plus, tous les composants soumis à la fois à des tensions élevées et des courants crête importants tels que thyratrons, condensateurs de décharge, résistance ballast, etc... sont placés dans une enceinte pressurisée et refroidie, elle même logée dans la partie supérieure du cylindre. On obtient ainsi une isolation électrique excellente, un niveau de parasites extérieurs très faible et une parfaite protection de l'utilisateur. Dans la partie basse du cylindre se trouve le système de circulation du gaz : ventilateurs et échangeurs.

Le volume de décharge est de $60 \mathrm{~cm} \times 3,5 \mathrm{~cm} \times 3,5 \mathrm{~cm}$. Comme dans le précédent laser, il est préionisé par une décharge Corona derrière la cathode semitransparente. Lue système de préionisation est déclenché de façon indépendante du circuit de décharge par un petit thyratron.

Le circuit de décharge est du type à transfert de charge, ce qui signifie qu'il comporte un banc de condensateurs primaires qui est chargé en continu. Au moment du tir laser, les charges stockées dans ces condensateurs primaires sont transférées par un thyratron de puissance dans le banc de condensateurs secondaires, lui-même connecté directement aux électrodes. L'inductance du circuit : condensateurs primaires thyratrons - condensateurs secondaires est assez élevée : $280 \mathrm{nH}$ de façon à limiter le courant thyratron. En revanche, l'inductance du deuxième circuit : condensateurs secondaires - décharge est, elle, aussi faible que possible : $6,7 \mathrm{nH}$ dans notre cas, de façon à permettre les courants crête élevés qui sónt nécessaires pour l'obtention de l'inversion de population et pour l'optimisation du rendement (adaptation d'impédance).

Il est donc clair que, au moment du tir, seuls les condensateurs secondaires alimentent la décharge. Les capacités des deux bancs de condensateurs sont identiques: $183 \mathrm{nF}$. Les constantes de temps caractéristiques de tous ces circuits ont été déterminées de façon, à la fois à réduire les contraintes électriques sur les thyratrons et à assurer un fonctionnement stable à toute cadence de répétition jusqu'à $500 \mathrm{~Hz}$. En effet, on sait que, dans un laser photodéclenché, il est important que toute ionisation résiduelle du gaz ait disparu lorsqu'on applique le champ électrique.

Lors d'un fonctionnement à cadence de répétition relativement élevế, on a pu montrer récemment ${ }^{4}$ que, mềme si le gaz est complètement renouvelé par l'écoulement entre chaque tir, divers phénomènes comme, par exemple, la diffusion, font revenir des charges entre les électrodes et donc provoquent des auto-amorçages nuisibles à l'effet laser. Un temps de montée long, même s'il favorise l'attachement des électrons sur $\mathrm{HCl}$, ne suffit pas à résoudre le problème. Dans des conditions usuelles rencontrées sur un laser XeCl, un temps de montée modérément court (environ $500 \mathrm{~ns}$ ) et un choix judicieux de l'instant de préionisation, permettent de contrôler correctement la décharge parce que l'avalanche provoquée par la préionisation démarre toujours avant celle qui pourrait être due à des charges résiduelles. Ceci ne pénalise nì l'efficacité du transfert de charge ni le rendement du laser et reste tout à fait acceptable pour le thyratron de puissance.

\section{RESULTATS EXPERIMENTAUX}

\subsection{Maquette 530}

Les performances de ce laser ont déjà été publiées : rappelons les brièvement : à faible cadence, l'énergie a atteint 1,4 J. A haute cadence de répétition, on a obtenu une 


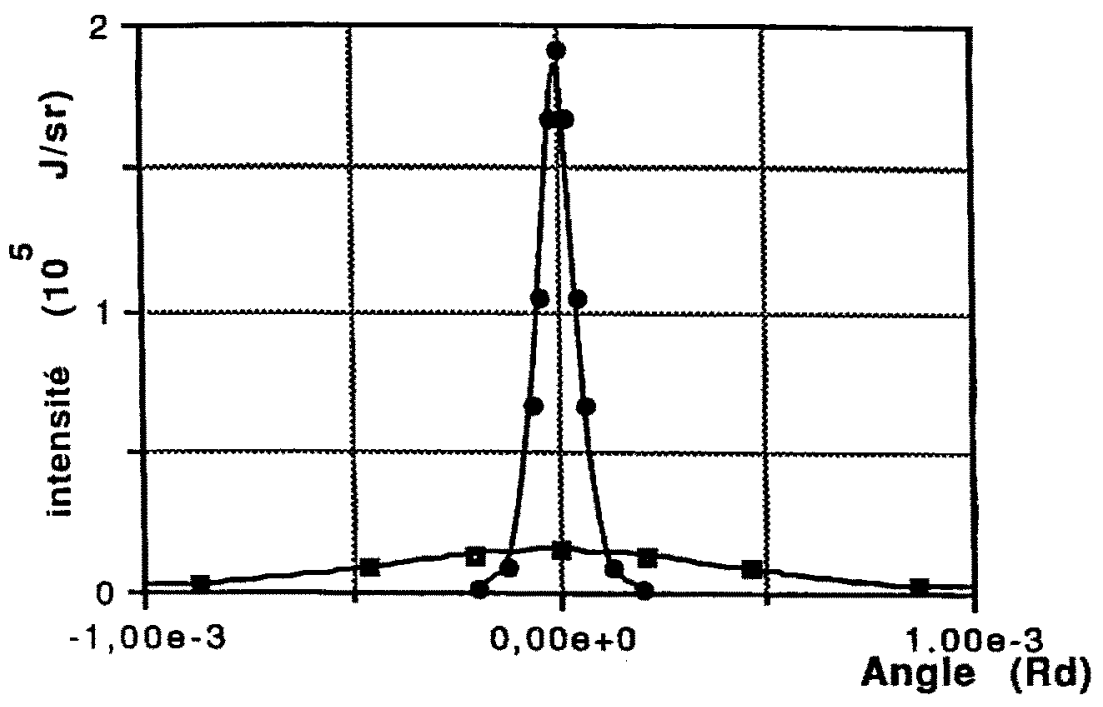

Figure 3 : Divergence du laser 530 en cavité stable (D) et instable (๑)

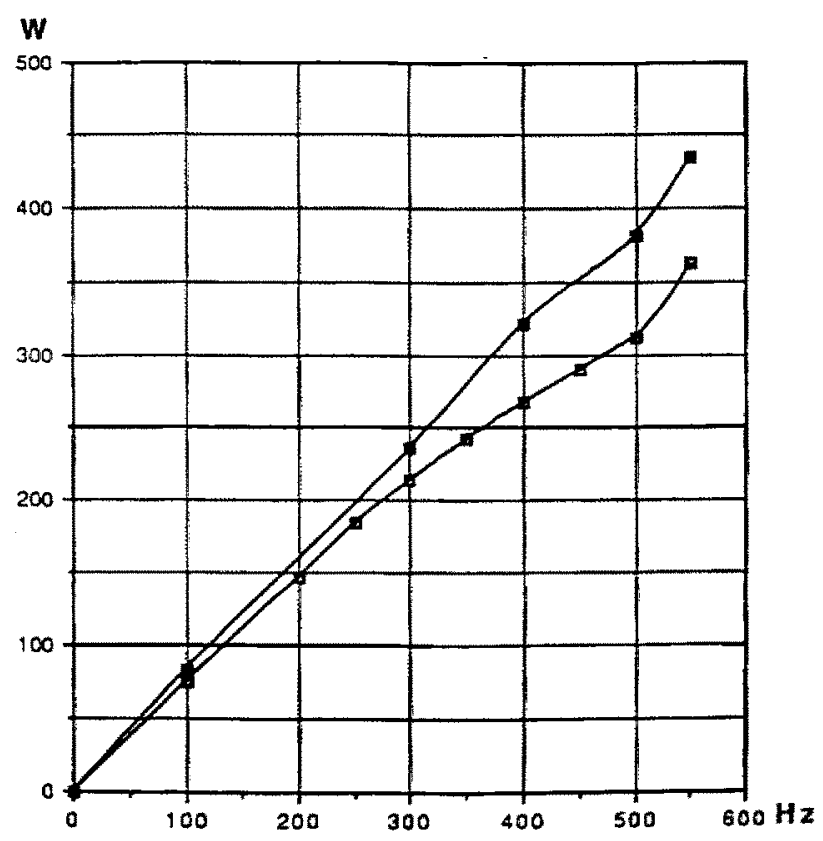

Figure 4: Laser 530 : puissance moyenne en cavité stable ( $\mathbf{\square})$ et instable ( $\square$ ) en fonction de la fréquence 
puissance moyenne de $500 \mathrm{~W}$ à $650 \mathrm{~Hz}$. La cadence de répétition maximum était de $750 \mathrm{~Hz}$.

Plus récemment, nous avons fait fonctionner ce laser en cavité instable toujours avec XeCl. Une cavité de grandissement 2,9 a été utilisée, le miroir avant consistant en un ménisque traité $R_{\max }$ sur un carré de $1 \mathrm{~cm}$ de côté en son centre.

La figure 3 montre l'amélioration de brillance apportée par cette cavité par rapport à une cavité "plan $R_{\max }$ ", "plan non traité". Quand à la figure 4, elle permet de constater que la diminution de puissance moyenne avec cette cavité instable est très faible.

Nous n'avons pas pu suivre l'évolution de la brillance avec la cadence de répétition mais un test simple : la production d'un plasma dans l'air au foyer d'une lentille semble indiquer que la baisse de brillance entre $1 \mathrm{~Hz}$ et $450 \mathrm{~Hz}$ reste inférieure à $20 \%$.

\subsection{Prototype 635}

Les performances à faible cadence de répétition de ce prototype sont représentées sur la figure 5 en fonction de la tension continue de charge. Sur cette figure, nous appelons rendement intrinsèque le rapport de l'énergie laser à l'énergie stockée dans les condensateurs secondaires lorsque la décharge s'allume, et rendement à la prise le rapport de l'énergie laser à l'énergie stockée dans les condensateurs primaires avant le tir.

Le rendement intrinsèque est caractéristique de la bonne qualité de la décharge et de l'efficacité du pompage. On constate qu'il est remarquablement élevé : plus de $4 \%$ pour un mélange $\mathrm{HCl}: 3$ mbars, Xe : 10 mbars, $\mathrm{Ne}: 4500$ mbars. Ceci est sans doute dû à la très bonne uniformité du champ électrique dans la zone de décharge et à la faible inductance du circuit de décharge qui nous permet de nous approcher de l'adaptation d'impédance entre le circuit et la décharge. Les valeurs de rendement que nous avons obtenues sont supérieures ou égales à celles que l'on peut obtenir avec des circuits à double décharge autrement appelés "spiker-sustainer" 5 .

Le rendement à la prise est lui, plus utile à l'utilisateur. S'il n'est pas aussi élevé que le précédent, c'est pour deux raisons :

- d'une part, un circuit de transfert de charge rapide est toujours un peu résistif, ce qui occasionne des pertes d'énergie,

- d'autre part, comme nous appliquons la préionisation toujours au même instant, la décharge ne se produit pas toujours exactement à la fỉn du transfert de charge.

Lors du fonctionnement à haute cadence, nous avons utilisé les mêmes conditions expérimentales de base avec, bien entendu, un écoulement rapide du gaz entre les électrodes (vitesse : $35 \mathrm{~m} / \mathrm{s}$ ).

Après ajustement de la largeur de la veine de gaz de façon à renouveler vraiment tout le volume de décharge, nous avons obtenu la courbe de la figure 6. Jusqu'à $300 \mathrm{~Hz}$, il s'agit pratiquement d'une droite qui correspond à une énergie de $1,8 \mathrm{~J} / \mathrm{tir}$, au-delà, la courbe rejoint une droite correspondant à $1,5 \mathrm{~J} / \mathrm{tir}$. Nous pensons, d'après l'expérience de la maquette 530 , dont les fréquences propres acoustiques sont sensiblement les mêmes, que la transition entre ces deux droites est due à l'apparition 


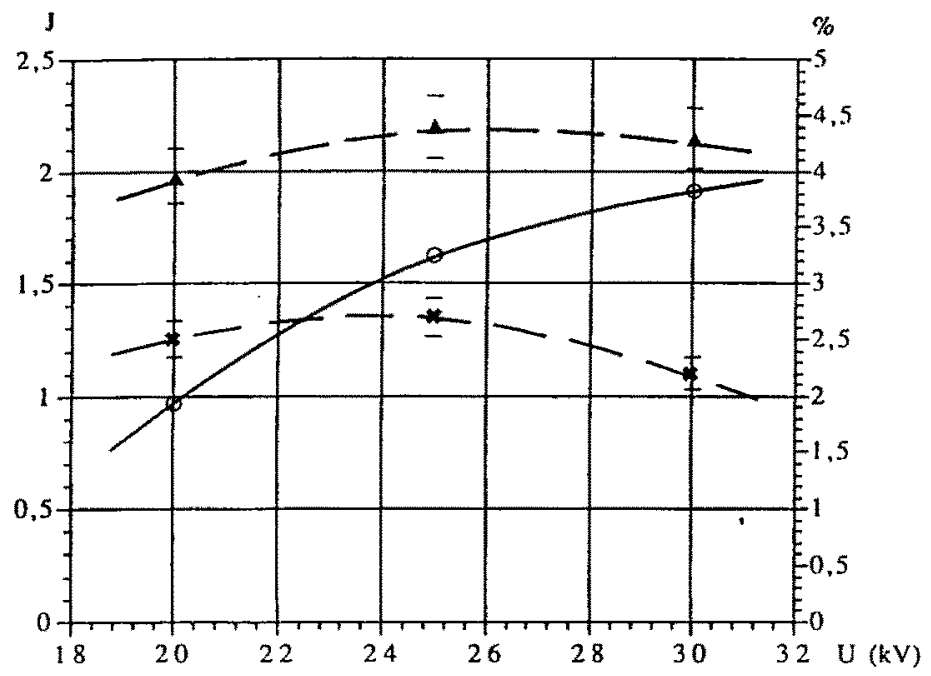

Figure 5: Laser 635 : énergie laser $(O)$, rendement intrinsèque $(\mathbf{A})$ et rendement à la prise $(X)$ en fonction de la tension de charge. $\mathrm{HCl}: 3 \mathrm{mbar}, \mathrm{Xe}: 10 \mathrm{mbar}$, $\mathrm{Ne}: 4500$ mbar.

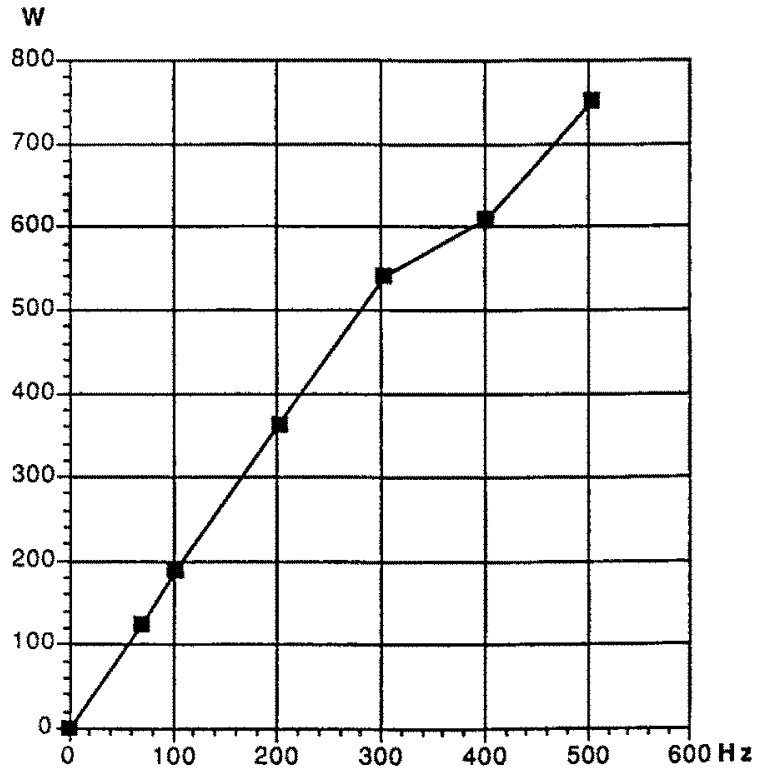

Figure 6: Laser 635 : puissance moyenne en fonction du taux de répétition. Tension de charge : $30 \mathrm{kV}$ 
d'un régime acoustique. Ceci est confirmé par l'observation des rafales à l'oscilloscope. Alors que, en-dessous de $300 \mathrm{~Hz}$, l'énergie par tir est tout à fait stable avec le temps, au-delà, on constate une décroissance initiale qui est suivie d'une stabilisation de l'énergie par tir. Cette décroissance peut être corrélée avec l'établissement d'un régime de réverbération acoustique dans l'enceinte du laser 6 .

\section{CONCLUSION}

Nous avons montré qu'il est possible de réaliser un laser $\mathrm{XeCl}$ photodéclenché fonctionnant à haute cadence de répétition (jusqu'à $700 \mathrm{~Hz}$ ) et à haute puissance moyenne (jusqu'à $750 \mathrm{~W}$ ).

Nous avons montré qu'un tel laser peut avoir un rendement intrinsèque comparable, voire même supérieur, à celui des lasers à excimères à double décharge qui utilisent des circuits beaucoup plus compliqués. Ceci a été obtenu grâce, en particulier, à une géométrie de tête laser particulièrement élaborée.

Nous avons réalisé un prototype industriel compact, potentiellement fiable, capable de fournir une puissance moyenne de $500 \mathrm{~W}$ à $500 \mathrm{~Hz}$, éventuellement avec une divergence de l'ordre de 5 fois la limite de diffraction.

\section{REMERCIEMENTS}

Cette étude a été réalisée avec les soutiens du MRT et du MICE dans le cadre du projet EUREKA EU 205.

\section{REFERENCES}

1) O. de WITTE, B. LACOUR et C. VANNIER

"Switchless TE gas lasers", Conference on Lasers and Electro Optics, Phoenix AZ, USA, Avril 1982

2) B. LACOUR et al

$9^{\text {th }}$ Symposium on Gas Flow and Chemical Lasers, Crête, Septembre 1992, SPIE, vol. 1810 , pp. 368-371

3) H. BRUNET et al.

AIAA, 24th Plasma Dynamics and Lasers Conference, Orlando, FL, USA, Juillet 1993

4) H. BESAUCELE

"Les auto-amorçages dans un laser à excimères photodéclenché de forte puissance", thèse de Doctotat de l'Université Louis Pasteur, Strasbourg 1, Mai 1994

5) W. M. LONG, M.J. PLUMMER et E.A. STAPPAERTS

Appl. Phys. Lett., vol. 43, p. 735, 1983.

6) J.P. TRONG

"Contribution à l'étude du fonctionnement quasi permanent d' un laser à excimère de puissance moyenne élevée" - Thèse de Doctorat de l'Université d'Aix Marseille II, Mars 1993. 